\title{
Notarinnen in der DDR
}

Prof. Dr. Gisela Shaw

University of the West of England, Bristol

\section{Zum Thema}

Mit einem Forschungsprojekt zum Thema „DDR-Notarinnen" betritt man weitgehend Neuland. Auch in Daniel Osterburgs kenntnisreicher und bisher einziger detaillierter Analyse des Notariats der DDR bleibt der Beitrag der Frauen (weitaus die Mehrheit) von marginaler und statistischer Bedeutung. ${ }^{1}$ Am 10. Oktober 1990, also eine Woche nach der offiziellen Vereinigung der beiden deutschen Staaten, waren fast 70 Prozent des gesamten DDR-Notariats Frauen, je verschieden verteilt über die 14 Bezirke (zwischen $100 \%$ in Schwerin und $48,1 \%$ in Potsdam). ${ }^{2}$

Hier soll gefragt werden: Wie kam es zu der Feminisierung des Notariats in der DDR? Welches war der ideologische, gesetzgeberische und organisatorische Rahmen? Wer waren die Frauen, die in dieser Institution arbeiteten? Wie erlebten sie ihre Arbeit? ${ }^{3}$

\section{Gesetzgeberischer und organisatorischer Rahmen 4}

Nach der Kapitulation Deutschlands im Mai 1945 blieb es im Notariat der sowjetisch besetzten Zone vorerst mehr oder weniger beim Status quo, das heißt bei der Institution des in preußischen Landesteilen üblichen Anwaltsnotariats. Dessen Entnazifizierung wurde von den dafür verantwortlichen Ländern und Provinzen nicht nur unterschiedlich, sondern auch weit toleranter gehandhabt als für Jurist/inn/en im öffentlichen Dienst - von Hilde Benjamin rückblickend als „unzureichende Entnazifizierung“ einer zudem überalterten fast ausschließlich aus bürgerlichen Schichten stammenden Berufsgruppe getadelt. ${ }^{5}$ Frauen im Anwaltsnotariat waren aufgrund der systematischen Diskriminierung während des Dritten Reichs eine verschwindende Minderheit.

Im Zuge der umfassenden Verwaltungs- und Justizreform von 1952 wurde die freiwillige Gerichtsbarkeit und damit die Institution des freiberuflichen Notariats aufgelöst und ein Großteil ihrer nun in zentral gelenkte Verwaltungstätigkeit umfunktionierten Aufgaben auf das nach sowjetischem Vorbild neu geschaffene staatliche Notariat übertragen. ${ }^{6}$ An jedem Kreisgericht wurde ein solches Notariat eingerichtet. Es unterstand dem jeweiligen Bezirksgericht und letztlich dem Ministerium der Justiz. ${ }^{7}$

Die Beschlüsse der Parteitage wurden verbindliche Grundlage notarieller Tätigkeit. Letztere sollte darin bestehen, „im gesamten Bereich des zivilen Rechtsverkehrs die Aufgabe der Sicherung der demokratischen Gesetzlichkeit zu übernehmen“ und insbesondere „darüber zu wachen, daß die Ziele der Politik der Regierung nicht verletzt werden und daß keine Verstöße gegen gesetzliche Bestimmungen begangen werden". ${ }^{8}$ Neben die fachlichen Aufgaben - Beglaubigungen und Beurkundungen, Nachlass- und Erbschaftssachen sowie
Pflegschaftssachen - traten damit politisch-gesellschaftliche Pflichten, von hervorragender Bedeutung bei allen Personalbeurteilungen. Dazu zählte insbesondere die massenpolitische Arbeit ${ }^{9}$, später allgemein als „Öffentlichkeitsarbeit" bezeichnet, wie etwa "Justizaussprachen“, Sprechtage außerhalb der Diensträume und das Abfassen von Zeitungsartikeln.

Neben staatlichem Notariat durften bereits zugelassene Anwaltsnotar/inn/e/n weiterhin ihre Tätigkeit ausüben. ${ }^{10}$ Jedoch waren keine Neuernennungen vorgesehen, die Anwaltsnotar/inn/en belassenen Tätigkeitsbereiche waren stark eingeschränkt, und mit zunehmendem Druck schloss sich die Mehrheit letztlich einem Rechtsanwaltskollegium an, was automatisch den Verlust der Zulassung zum Notar mit sich brachte. ${ }^{11}$ Nach knapp zwei Jahrzehnten (1970) war die Zahl der noch aktiven Einzelnotar/inn/en insgesamt auf 42 geschrumpft. ${ }^{12}$ In Ostberlin galt die Regelung, dass alle Bestellungen von Notar/inn/en (und Rechtsanwält/inn/en) am 18. April 1953 ihre Gültigkeit verloren und Anträge auf Neuzulassungen vom Magistrat von Groß-Berlin (ab 1976 vom Justizminister) zu entscheiden waren. ${ }^{13}$ Tatsächlich wurden einige wenige solcher Anträge positiv entschieden, weil die

1 Das Notariat in der DDR - Aufgaben, Funktion, Ausbildung, Herkunft und Kontrolle der Notare, Köln 2004.

2 Bundesnotarkammer, Notarverzeichnis der Bundesländer Ost 1990. Dazu Shaw, Gisela, Juristinnen in den neuen Bundesländern, in: Zeitschrift für Rechtssoziologie, 2/1994, S. 191-207; dies., Window of Opportunity or Flash in the Pan? Women Notaries in the New Federal States of Germany, German Life and Letters, 4/1997, S. 557-573; auch in Littler, Margaret (Hg.), Gendering German Studies. New Perspectives on German Literature and Culture, Blackwell, 1997, S. 179-195.

3 Hauptinformationsquellen zur versuchsweisen Beantwortung dieser Fragen sind einmal Dokumente des Ministeriums der Justiz im Bundesarchiv in Berlin, zum andern halbstrukturierte Gespräche mit 25 Zeitzeuginnen aus den fünf neuen Bundesländern und Berlin, die zwischen September 1990 und (die Mehrheit) Februar/März 2007 geführt wurden. Ihnen allen sei an dieser Stelle für ihr engagiertes Interesse und ihre Gesprächsbereitschaft herzlich gedankt.

4 Dazu v. a. Osterburg; auch Benjamin, Hilde, Geschichte der Rechtspflege der DDR 1945-1949 (= Band I), Berlin (DDR) 1976, und Zur Geschichte der Rechtspflege der DDR 1949-1961, Berlin (DDR) 1980 (= Band II).

5 Benjamin I, S. 83.

6 Verordnung über die Errichtung und Tätigkeit des Staatlichen Notariats, DDR GBI. 1952, 1055 und Verordnung über die Übertragung der Angelegenheiten der freiwilligen Gerichtsbarkeit, DDR GBI. 1952, 1057. Beide 15. Oktober 1952.

7 Benjamin II, S. 135 .

8 Artzt, Werner (Hauptabteilungsleiter im Ministerium der Justiz), Die Ausgliederung der Freiwilligen Gerichtsbarkeit und die Errichtung des Staatlichen Notariats, Neue Justiz, 12/1952, S. 517-521; Zitat S. 521.

9 Benjamin II, S. 201.

10 Zum Anwaltsnotariat s. Brand, Peter-Andreas, Der Rechtsanwalt und der Anwaltsnotar in der DDR, Köln 1985, S. 147, und Osterburg, a.a.O., S. 209-224.

11 Anordnung über den Amtsbezirk der freiberuflichen Notare vom 22.1.1953, DDR GBI. 1953, 141.

12 BArch DP1 VA 2646 (Überprüfung der Tätigkeit der Einzelnotar/inn/ e/n).

13 Osterburg, a.a.O., S. 209. 
Expertise für den Umgang mit dem kapitalistischen Ausland erforderlich war. Mit Beglaubigungen und Beurkundungen für einfache Bürgerinnen und Bürger hatten sie jedoch nichts zu tun.

Höhepunkt und Abschluss der gesetzgeberischen Gestaltung des DDR-Notariatswesens bildete das Gesetz über das staatliche Notariat vom 11. Februar $1976^{14}$, die logische Folge der Einführung des Familiengesetzbuches, der neuen Zivilprozessordnung und des lang geplanten und diskutierten, am 19. Juni 1975 von der Volkskammer verabschiedeten Zivilgesetzbuches ${ }^{15}$, das an die Stelle des Bürgerlichen Gesetzbuches trat. 1990 zeigte sich, dass, wer seine Ausbildung noch vor 1975 abgeschlossen hatte, die Überleitung in die bundesdeutsche Gesetzgebung, insbesondere im Bereich des Handels- und des Gesellschaftsrechts, weit leichter bewältigte, als wer mit dem BGB nicht mehr vertraut war.

Die Verordnung über die Tätigkeit von Notaren in eigener Praxis vom 20. Juni 1990 bedeutete die Rückführung der freiwilligen Gerichtsbarkeit zu den ordentlichen Gerichten und die Einführung des Nur-Notariats in der DDR (mit Ausnahme Ostberlins, wo das Anwaltsnotariat bestehen blieb) - eine einmalige Chance, aber auch eine ungeheuere Herausforderung für diejenigen (die Mehrheit, dies wiederum vor allem Frauen), die sich für den Schritt in die Unabhängigkeit entschieden. ${ }^{16}$ So begann der fast märchenhaft anmutende unaufhaltsame Aufstieg des Notariats in der ehemaligen DDR vom Aschenputtel zur Prinzessin der ostdeutschen Justiz. ${ }^{17}$

\section{Kaderprofil im staatlichen Notariat}

Wie das Kaderprofil der Richterschaft sollte auch dasjenige des staatlichen Notariats den sozialistischen Anforderungen in Bezug auf sozialen Hintergrund und ideologische Einstellung und Qualifizierung entsprechen. Jedoch erwies sich dieses Ziel im staatlichen Notariat als unerreichbar, und wohl, so kann man schließen, politisch gesehen auch von geringerer Priorität. 1985 waren immer noch nur 63,4 Prozent aller staatlichen Notarinnen und Notare SED-Mitglied (Leiter: $72,3 \%$; sonstige Notar/inn/e/n: 54\%), bei den Richterinnen und Richtern am Kreisgericht dagegen 96,7 Prozent. ${ }^{18}$ An Einzelnotar/inn/en zeigte die Justizverwaltung wenig Interesse, wenngleich sie auch allmählich in die Revisionen einbezogen wurden.

Den (aus der Sicht der Partei) ideologischen Mängeln im staatlichen Notariat entsprach im Mikrobereich notarieller Arbeit ein in der Justiz sonst nicht vorhandener Grad an Selbstständigkeit und Eigenverantwortung. Gesprächspartnerinnen sprachen von einer "Nischenfunktion“ in der Justiz, einer „politischen Insel“.

„Natürlich war man Begrenzungen unterworfen. Wir durften z.B. nicht nach drüben schreiben. Alle Vierteljahr mussten wir unterschreiben, dass wir keine Westkontakte hatten. Wir wurden tüchtig geknebelt. Ich ging statt in die FDJ lieber in die Junge Gemeinde.... Aber man hat mir nie die Pistole auf die Brust gesetzt: Tritt in die Partei ein, sonst kannst du deinen Beruf nicht ausüben!" (U. R.)
Die unabhängige Arbeit, der persönliche Kontakt mit Bürgerinnen und Bürgern in politisch relativ geschützter Umgebung schuf, so meine Informantinnen, die Grundlage zu gegenseitigem Vertrauen, was für viele von ihnen wirtschaftliche Nachteile wettgemacht habe. Selten habe sich eine Notarin um eine Leitungsfunktion bemüht, da es dort vor allem um das Abfassen von Berichten und Statistiken gegangen sei und die politische Einstellung sehr viel schwerer gewogen habe.

„Es war eine relativ selbständige Arbeit. Man war niemandem weisungsunterstellt, was die Arbeit anging, nur disziplinarisch. Das einzige, was sie konnten, war, eine Sache an sich ziehen, nicht aber die Entscheidung selbst beeinflussen. Man machte die Arbeit gern. Bekam auch viel Anerkennung von den Leuten. Bei Anwälten ist das anders. Da verliert immer einer. Beim Notar gewinnen alle und sind in der Regel dankbar." (B. E.)

„Jeder Bürger hatte andere Problem und Sorgen. Das war das Interessante. Zu DDR-Zeiten gab es nur bestimmte Richtungen. Man musste sagen: So gebt's nicht, aber so können Sie's machen... man hat es immer versucht, das, was ihrem Interesse [dem Interesse der Bürger] am nächsten kam, irgendwie in die Bahn zu bringen. ... wenn das so Gespräche waren, die dann gegen die Staatspolitik gingen, Reisesachen usw., dann hat man ibnen gesagt: Das lassen wir lieber weg, darüber diskutieren wir nicht. Aber wir mussten nachher nicht zum Bezirksgericht melden: Der und der hat das und das geäußert. Also so war das nicht. Ich denk', das hat viel dazu [zum Vertrauen] beigetragen. Und beim Staatsanwalt war es so, wenn das da jemand geäußert hatte, dann wusste man am nächsten Tag, was los war. " (U. R.)

Jedoch ist zu bedenken, dass die Berufsauffassung meiner Gesprächspartnerinnen nicht unbedingt zu verallgemeinern ist. Denn ein Posten im staatlichen Notariat galt vielen Juraabsolvent/inn/en nur als Durchgangsstation, bis sich eine Richterstelle oder (noch begehrter, aber weit seltener) eine Stelle in einem Rechtanwaltskollektiv oder auch eine Justitiarstelle in der Wirtschaft fand. Oft war das Notareinkommen nur als Zweiteinkommen praktikabel.

Für das staatliche Notariat hatten SED-Programme von Anfang an die besondere Förderung der Frauen im Prozess der Demokratisierung der Justiz gefordert, anfangs im Hinblick auf Richter/innen und Staatsanwält/inn/e/n, nach 1952 auch für das Notariat - sowohl aus idealistischen wie auch aus ganz praktischen Gründen. ${ }^{19}$ Kaderanalysen seit den Sechzigerjahren geben stets Auskunft über Erfüllungsraten der Frauenförderungspläne.

Juristinnen und Juristen mit Hochschulqualifikationen standen dem staatlichen Notariat anfangs nicht zur Verfügung. Stattdessen entschied man sich für das Modell einer stufenwei-

\footnotetext{
14 Notariatsgesetz, GBI. I Nr. 6 S. 93.

15 GBI.I Nr. 27 S. 465

16 GBI. v. 30.6.1990 S. $475 \mathrm{ff}$.

17 Shaw, GLL 4/50, Okt. 1997, S. 557-573

18 BArch DP1 VA 4491 (Analyse des Kaderbestandes im Verantwortungsbereich des Ministeriums der Justiz, 24.2.1986).

19 Benjamin II, 92-3.
} 
sen Qualifizierung, wobei politisch-ideologische Qualifikationen vor akademischen Vorrang hatten. ${ }^{20} \mathrm{Ab} 1952$ wurden in Ettersburg bei Weimar drei Notarlehrgänge abgehalten. Die Auswahl der Teilnehmer/innen wurde vor allem nach sozialem Hintergrund und politischer Orientierung bestimmt. So dominierten Teilnehmer/innen mit bescheidener Schulbildung und einfachen praktischen Berufsqualifikationen, darunter zunehmend Frauen. Der angestrebte Frauenanteil von 50 Prozent wurde allerdings nicht erreicht. ${ }^{21}$ Zielvorgabe (aber keineswegs zwingend gefordert und durchgeführt) war dann eine juristische Weiterqualifizierung durch Fernstudium, das seit 1958/59 auch für Notarinnen und Notare zugänglich wurde und neben der notariellen Tätigkeit herlief. Für Frauen, die mehrheitlich bereits Familienpflichten hatten, war dies ein anstrengendes Pensum, das auch in späteren Jahren prägend blieb.

Personalakten ausgeschiedener Notarinnen lassen erahnen, mit welchen Schwierigkeiten besonders die in der Anfangsphase ernannten Notarinnen zu kämpfen hatten, um Beruf und Familie unter einen Hut zu bringen, selbst wenn sie kein Jurastudium in Angriff nahmen. Hier nur ein Beispiel - ein Auszug aus dem Brief einer Notarin an ihre Justizverwaltungsstelle aus dem Jahr 1963 mit der Bitte um Entpflichtung:

„Durch die schlechte Fabrtverbindung zwischen meinem Wohnort (ich bin täglich fast 13 Stunden unterwegs) und dadurch, dass in K. keinerlei Aussicht auf Zuweisung einer Wohnung besteht, bin ich aus gesundheitlichen Gründen, da ich Schilddrüsen- und herzkrank bin, nicht mehr in der Lage, den Dienst beim Staatlichen Notariat K. zu erledigen. Ich habe meine Mutter, die gelähmte Beine hat und sich sehr mühsam nur im Hause mit Hilfe eines Stubles bewegen kann, zu versorgen. Ausserdem habe ich einen vierjährigen Pflegesohn, den ich versorgen muss. Während der Heizperiode habe ich ibn morgens schon um $6 \mathrm{Ubr}$ in den Kindergarten bringen können, da die Reinigungskraft ab 6 Ubr früh die Heizung versorgte. Nach der Heizperiode beginnt der Kindergarten, wie auch alle anderen Kindergärten ..., frühestens um 6.30 Uhr. Der Bus nach K. faehrt aber bereits um 6.10 Uhr.

Abends bin ich zwischen 18.30 und $19.00 \mathrm{Uhr}$ im Hause und muss dann noch alle Hausarbeiten usw. erledigen. ... Durch die oben angeführten Gründe war ich auch nicht in der Lage, in K. abends massenpolitische Arbeit zu leisten. Dieser Zustand kann mich nicht befriedigen. "22

Seit Mitte der Sechzigerjahre stieg der Anteil der Absolvent/ inn/en juristischer Staatsexamen am Notariat stetig, zur Zeit der Wende betrug er etwa 75 Prozent. Parallel dazu wuchs der Frauenanteil sowohl an der juristischen Studentenschaft als auch am Notariat. So verzeichneten die Universitäten Jena und Berlin, die Hauptausbildungsstätten für Jurist/inn/en, von 1972 auf 1974 einen Anstieg des Anteils weiblicher Studenten von 44,2 Prozent auf 73,1 Prozent. $^{23}$

Der chronische Personalmangel im staatlichen Notariat - belegt in detaillierten, nach ministeriell vorgegebenen Kriterien abgefassten Kaderanalysen der Bezirksgerichte - erschwerte das Anlegen strikter Qualitätsmaßstäbe. Dazu kam die beträchtliche und mit dem stetigen Anstieg des Frauen- anteils zunehmende Fehlquote, verursacht durch Krankheit, Haushaltstag, Schwangerschaftsurlaub, kranke Kinder und Freistellungen aller Art. Ein Bericht über die Kaderlage in der Justiz nach der Richterwahl von $1965^{24}$ meldete insgesamt 25 unbesetzte Planstellen im staatlichen Notariat (bei einem Plansoll von 421), dazu 93 Notarinnen und Notare im juristischen Fernstudium. Für die Kollegen führe das zu oft unzumutbarer Arbeitsbelastung, insbesondere in den zahlreichen kleinen Dienststellen mit nur ein oder zwei Planstellen. Im Januar 1983 verzeichnete das Staatliche Notariat Berlin ca. 34 Prozent durch Krankheit oder Freistellung abwesende Kolleg/ inn/en. ${ }^{25}$ Zeitweise waren dort nur 52 Prozent der Planstellen besetzt. ${ }^{26} 25$ bis 30 Prozent scheinen, vor allem in der Spätzeit der DDR, als Norm gegolten zu haben.

Das Durchschnittsalter der Notare lag bis zum Ende der DDR unter dem der übrigen Justizangehörigen, wenngleich es im Laufe der Jahre allmählich gestiegen war. Zumindest zwei Gründe lassen sich erkennen. Einmal begann die systematische Ausbildung staatlicher Notar/inn/e/n in der Entwicklung der DDR später als die von Richter/inne/n und Staatsanwält/inn/ en. Zum andern war das Mindestalter für eine Richterlaufbahn 25 Jahre, während es für Notar/inn/e/n keine Mindestgrenze gab. Juristische Examina waren oft bereits mit 22 oder 23 Jahren abgeschlossen, und Frauen, die keinen Wehrdienst abzuleisten hatten, hätten für eine Richterstelle zwei oder drei Jahre mit einer weiteren Ausbildung oder Tätigkeit überbrücken müssen. So war die Wahrscheinlichkeit eines geringeren Durchschnittsalters in der Justiz desto größer, je größer der Frauenanteil in der jeweiligen Berufsgruppe war. 1975 lag der Anteil der Unter-Dreißigjährigen bei Notarinnen bei 39,1 Prozent (Richterinnen: 26,9\%; Staatsanwältinnen: 26,6\%).27 Frauenförderung, die Entwicklung und Planung weiblicher Reservekader auf allen Ebenen und die Erhöhung des Frauenanteils in leitenden Funktionen in der Justiz blieb bis zum Ende der DDR eine der Prioritäten bei der Kaderplanung des Ministeriums der Justiz.

Im Anwaltsnotariat waren und blieben Frauen die Ausnahme. Eine Personalaufstellung aus den frühen 1950er-Jahren für Rechtsanwält/inn/en und Notar/inn/en in ausgewählten Bezirken nennt im Bezirk Dresden sechs Frauen (von 69) (darunter Margarete Bürger, geb. 1902; Dr. Maria Cordes, geb. 1905; und Dr. Irene Menzel, geb. 1905); im Bezirk Leipzig unter den 59 Anwaltsnotaren keine Frau; zwei Frauen von 19 im Bezirk Cottbus (Käte Fechner; Dr. Ingeborg Fischer, geb. 1905). ${ }^{28}$ Im Jahr 1970 gab es in der DDR insgesamt nur noch

\footnotetext{
20 Benjamin II, S. 134.

21 Dazu Osterburg, a.a.O., S. 129 und 180 f.

22 U. a. BArch DP1 SE 30931 (Personalakten ausgeschiedener Notare).

23 Maser, Willi/Scholz, Erhard, Entwicklung und Förderung der Frauen in den Justizorganen der DDR, Neue Justiz 1975, S. $562 \mathrm{ff}$.

24 BArch DP1 SE 2167, Bd. 2 (Kaderlage in den Justizorganen 1966).

25 BArch DP1 VA 4331 (Einschätzung der Lage im StN Berlin unter besonderer Berücksichtigung des Leistungsstandes der Kader).

26 Gespräch mit Sabine Herrmann, Berlin, 9.5.2007.

27 Osterburg, loc. cit., S. 181.

28 BArch DP1 SE 2398 1-2 (Rechtsanwälte und Notare in den Bezirken 1952-55).
} 
47 Einzelnotar/inn/e/n, darunter fünf Frauen: Drei im Bezirk Dresden (die oben namentlich Genannten) sowie Dr. Liselotte Kottler (geb. 1909) in Suhl und Dr. Ingeburg Gentz (geb. 1904) in Berlin. ${ }^{29}$ Das Anwaltsverzeichnis der DDR für 1990 schließlich dokumentiert noch zwei Anwaltsnotarinnen, nämlich Dr. Maria Cordes und Dr. Liselotte Kottler, ${ }^{30}$ wobei deren Arbeitsschwerpunkt immer ihre anwaltliche Tätigkeit war. ${ }^{31}$

\section{Einzelprofile}

Bei Gesprächen mit ehemaligen staatlichen Notarinnen war eine meiner wiederkehrenden Fragen: „Gab es in der DDR herausragende Notarinnen? “ Bald wurde offensichtlich, dass diese kleine, über das ganze Land verstreute, stark feminisierte und in der Justizhierarchie ganz unten rangierende Berufsgruppe im allgemeinen wenig geeignet war, herausragende Persönlichkeiten hervorzubringen. Jedoch begegneten mir zwei Namen, denen ich beschloss nachzugehen: Dr. Ingeburg Gentz und Sabine Herrmann. Erstere eine noch vor dem 2. Weltkrieg ausgebildete politisch links orientierte Berliner Anwaltsnotarin, die auch in der DDR ihren Status als Einzelanwältin und -notarin aufrecht erhielt und zudem bis in die 1970er-Jahre eine Schlüsselfigur für die Devisenwirtschaft der kommunistischen Partei darstellte. Letztere eine in der DDR ausgebildete Juristin der nächstjüngeren Generation, die es innerhalb des staatlichen Notariats der DDR zu Einfluss und Ansehen brachte.

\section{Dr. Ingeburg Gentz}

Geboren 1904 in Potsdam, besuchte sie von 1913 bis 1924 das dortige Lyzeum und Oberlyzeum und bestand dort 1924 die Abschlussprüfung als Lehrerin sowie im Herbst desselben Jahres (als Gastschülerin) in Kiel die Reifeprüfung am humanistischen Gymnasium. ${ }^{32}$ Die nächsten Etappen waren: 1924 28 Studium der Rechtswissenschaften in Kiel. 1928 Referendarexamen und Heirat mit dem damaligen Justizrat (späteren Ministerialrat) Dr. Werner Gentz (geb. 1884). 1929 bzw. 1931 Geburt der Söhne Jürgen und Ulrich. 1933 Assessorexamen mit „vollbefriedigend“ bestanden. 1933-35 Kampf vor einem Ehrengericht um ihre Zulassung als Rechtsanwältin - ihre politische Haltung sowie ihre Eigenschaft als Hausfrau standen im Wege. ${ }^{33}$ Nach gewonnenem Verfahren konnte sie endlich ihre Berufstätigkeit aufnehmen. Von 1935 bis 1945 arbeitete sie als Rechtsanwältin und Notarin in ihrem Büro in BerlinZehlendorf.

Nach Kriegsende bemühte sich Ingeburg Gentz sofort um ihre erneute Zulassung. Am 3. Juni 1945 schrieb sie an den Präsidenten des Stadtgerichts Berlin:

„Seit 1935 übe ich den Anwaltsberuf aus. Ich habe mich in dieser Zeit stets für politisch Verfolgte und für Juden eingesetzt, habe Antifaschisten verteidigt, soweit ich vom Volksgerichtshof nicht abgelehnt wurde, und habe insbesondere erreicht, dass der bekannte antifaschistische Schriftsteller Ernst Nikisch, der vom Volksgerichtshof zu lebenslänglischem Zuchthaus verurteilt worden war, nicht der ihm wiederholt drohenden „Liquidierung“ durch die SS zum Opfer fiel. In meiner Praxis werden mehrere hundert Pflegschaften und Vormundschaften der Amtsgerichte Charlottenburg und Schöneberg, insbesondere auch jüdischer Vermögensmassen, die ich bisher zu einem guten Teil vor dem Zugriff des Finanzamtes retten konnte, bearbeitet, die sämtlich eine anwaltiche Betreuung notwendig machen."

Bereits am 3. Juli 1945 bekam Ingeburg Gentz bei allen Amtsgerichten sowie beim Stadtgericht Berlin ihre (zunächst vorläufige, 1947 endgültige) Zulassung als Rechtsanwältin, und am 5. September desselben Jahres die (ebenfalls zunächst vorläufige) Bestellung zur Notarin, letztere für den Bezirk des Stadtgerichts mit Amtssitz in Berlin-Schöneberg.

Damit begann die zweite, nicht weniger spannungsreiche und auf's engste mit der politischen Entwicklung in Deutschland verquickte Phase ihrer beruflichen Laufbahn, diesmal bedingt durch die Wirren und Ironien der deutschen Teilung. Mit der politischen Spaltung Deutschlands ergab sich auch die allmähliche Spaltung der deutschen Justiz, was Gentz unmittelbar betraf. Denn nachdem sie ursprünglich ihre Anwaltszulassung durch die Westlichen Alliierten für ganz Berlin bekommen hatte, erwarb sie 1948 zusätzlich die Zulassung beim Antsgericht Teltow und dem Landgericht Potsdam sowie eine Notarbestellung, da die DDR-Regierung die Vorschrift der Rechtsanwaltsordnung ( $\mathbb{2} 26 \mathrm{RAO}$ ), nach der jede/r bei einem deutschen Gericht zugelassene Anwältin oder Anwalt vor jedem deutschen Gericht auftreten durfte, für ihre Gerichte nicht anerkannte. So unterhielt Ingeburg Gentz 1948 bis 1950 gleichzeitig zwei Büros, eins in Ostberlin in Kleinmachnow (ihrem neuen Wohnort) und eins in Westberlin. Zum zweiten Mal in ihrem Leben wurde sie daraufhin 1951 vor ein Disziplinargericht der Rechtsanwaltskammer zitiert, diesmal initiiert vom Generalstaatsanwalt in BerlinWilmersdorf, dem sie aber nicht Folge leistete. Ihre Rechtfertigung: Durch die politische Spaltung seien zwei getrennte Gerichtsbezirke entstanden, und die Westberliner Staatsanwaltschaft sei für Rechtsanwältinnen und Rechtsanwälte im östlichen Sektor nicht zuständig. Wieder wurde sie letztlich freigesprochen. Das Ehrengericht befand, die Justiz habe es versäumt, die Konsequenzen aus der veränderten faktischen politischen Lage zu ziehen und könne für dieses Versäumnis nicht Einzelne verantwortlich machen.

29 BArch DP1 VA 2646 (Überprüfung der Tätigkeit der Einzelnotare [1970]).

30 DP1 SE 10, Nr. 3.

31 S. dazu Röwekamp, Marion, Juristinnen - Lexikon zu Leben und Werk, Baden-Baden 2005.

32 Die im Folgenden zusammen getragenen Informationen stammen mehrheitlich aus drei Quellen: Dokumenten im Bundesarchiv Berlin-Lichterfelde (BArch DP1 VA 3561 Dokumente zur Tätigkeit des Büros Gentz, 1974-76; BArch DP1 SE 3100 Personalakten ausgeschiedener Rechtsanwälte/Notare); Gesprächen mit ehemaligen staatlichen Notarinnen in Berlin im Frühjahr 2007, insbesondere Notarin a.D. Sabine Herrmann am 9.5.2007; sowie verstreuten Anmerkungen in Osterburg, Daniel, op. cit. Bedauerlicherweise stand die als Anwaltsnotarin in Berlin praktizierende Enkelin Ute Gentz nicht für ein Gespräch zur Verfügung.

33 BArch DP1 SE 3100 Personalakten ausgeschiedener Rechtsanwälte/ Notare. 
So gehörte Ingeburg Gentz denn zu den wenigen in beiden Teilen Berlins zugelassenen Anwaltsnotar/inn/en und gelangte dadurch in eine exponierte Stellung. 1946 beurkundete sie beim Zusammenschluss von SPD und KPD die Umwandlung des gesamten Parteivermögens in eine $\mathrm{GmbH}$, später dann auch die Umwandlung der Parteibetriebe von GmbHs in „organisationseigene Betriebe“. 1947-49 gehörte sie dem Präsidium der Berliner Rechtsanwaltskammer an, was es ihr erlaubte, der Heranziehung zur Richterin zu entgehen. In den folgenden drei Jahrzehnten wurde sie für die DDR zur unentbehrlichen Vermittlerin im Umgang mit dem Westen. Sie betreute DDR-Urkunden, die im westlichen Ausland verwendet werden sollten, und brachte mit ihrer juristischen Arbeit erhebliche Devisenbeträge aus kapitalistischen Ländern ein, die sie persönlich in die DDR einführte und bei der Industrie- und Handelsbank ablieferte. Dabei ging es vor allem um ausländische Nachlässe, die Registrierung von Patenten und Schutzrechten im kapitalistischen Ausland und die Erledigung von Aufträgen des sowjetischen Konsulats in der DDR und des Konsulats in Westberlin. In vielen kapitalistischen Ländern hatte sie „Verbindungsanwälte mit fortschrittlicher Einstellung“, über die sie Angelegenheiten im Interesse der DDR und der Sowjetunion regelte. Das Protokoll einer offiziellen „Aussprache“ stellte fest:

„Ihre größten Verdienste bestehen darin, dass sie in der Lage ist, nicht nur Forderungen durchzusetzen und Devisen einzubringen, sondern dass sie die Devisen auch direkt in die DDR einführt und bei der IHB [Industrie- und Handelsbank] abliefert. Da manche Zahlungen ausländischer Banken nur in Raten erfolgen oder es sich um monatliche Rentenzahlungen handelt, erfolgen die Gutschriften auf ibren Konten bei westberliner Banken. Von dort hebt sie die Beträge (monatlich bis 1.000 DM) ab und bringt sie in die DDR. ... In den USA werden die Zablungen von Dollar z. $Z$ t. auch davon abhängig gemacht, dass USA-Gerichte prüfen, ob die Dollarbeträge in der DDR von Erben verwandt werden können. ... Wenn ein Prozess notwendig wird, gibt sie die Sache an das Büro Hofmann ab oder bedient sich eines Rechtsanwalts im betreffenden Staat, in dem der Prozess zu fübren ist. ... Genn. Gentz hat enge Kontakte zu den Inur-Kollegien in Moskau und Budapest und mit fortschrittlichen Rechtsanwälten in den USA, Frankreich, Schweiz und der BRD und ist oft in Moskau, Budapest, Paris und Frankfurt a. M. "34

In einem Vermerk vom 11. Januar 1974 bestätigte der Minister für Außenhandel, dass das Büro Gentz 10 bis 20 Prozent des Gesamtaufkommens der in der Realisierung von Nachlässen und anderen Forderungen im kapitalistischen Ausland erzielten Deviseneinnahmen erbracht habe.

Zur Erleichterung ihrer grenzüberschreitenden Tätigkeit bekam sie seit dem Bau der Mauer laufend Passierscheine für die Staatsgrenze, anfangs auf monatlichen Antrag beim Präsidium der Volkspolizei, dann ab Juni 1963 in Form einer Global-Genehmigung des Ministers des Inneren, nachdem sich der Leiter der Justizverwaltungsstelle in einem Schrei- ben vom 4. Mai 1962 an das Ministerium der Justiz persönlich für die umgehende Bewilligung ihres Antrags eingesetzt hatte:

„Sie ist der einzige Notar, der aufgrund früherer Bestimmungen zu notariellen Handlungen vor Institutionen der westlichen Besatzungsmächte berechtigt ist und der in der Lage ist, sehr diffizile Aufgaben für unser Grosshandelsunternehmen in Westberlin zu erledigen. "35

Ab 1967 wurde diese Genehmigung auf den Sohn Dr. Jürgen Gentz ausgedehnt, der als Notarvertreter im Büro seiner Mutter arbeitete. Jedoch geriet mit der internationalen Anerkennung der DDR 1971 diese Sonderlösung für das Büro Gentz ins Wanken. Spannungen zwischen den zuständigen Ministerien (Inneres, Justiz und Außenhandel) traten deutlich zutage. Hauptgegner der Fortdauer des Status quo war der Minister der Justiz. Ihm lag daran, endlich die vom Büro Gentz in Eigenregie unternommenen und vom Minister für Außenhandel sanktionierten Tätigkeiten unter seine Kontrolle zu bekommen, zumal man ihn bisher im Zusammenhang mit den Westreisen von Ingeburg und Jürgen Gentz noch nicht einmal um Genehmigung oder Befürwortung befragt hatte. Sein Plan war, diese Tätigkeiten dem Rechtsanwaltsbüro für Internationale Zivilrechtsvertretungen zu übertragen. Zunehmend sahen sich Mutter und Sohn Gentz durch zentrale Anweisungen behindert.

Eine Aussprache zwischen dem Ministerium und Ingeburg Gentz sollte „den Charakter und Umfang der Tätigkeit der Rechtsanwälte Gentz in den nichtsozialistischen Staaten einsschließlich Westberlin feststellen “ und Klarheit darüber schaffen, dass

- „die zuständigen zentralen Organe über Umfang und Art dieser Tätigkeit zur Gewährleistung eines einheitlichen abgestimmten Verfahrens einen entsprechenden Überblick haben müssen,

- künftig keine Legalisationen notarieller Urkunden beim Präsidenten des Landgerichts Berlin mehr zu erfolgen haben sondern hierfür ausschliesslich das Ministerium der Justiz zuständig ist,

- keine pauschalen Ausreisegenehmigungen mehr für Westberlin und die BRD erteilt werden, sondern nur noch im Einzelfall nach vorheriger schriftlich begründeter Antragsstellung."

Genn. Gentz sei „sichtlich erregt“ gewesen, berichtet das Protokoll. ${ }^{36}$ Sie habe nicht akzeptieren wollen, dass die BRD nun für die DDR Ausland sei. In dem Gespräch von 11/2 Stunden habe sie auf Anfrage einen allgemeinen Überblick über die Tätigkeit ihres Büros gegeben, jedoch konkrete Fragen „weitausholend, weitschweifig und unübersichtlich“ dargestellt. Bei der Diskussion über die Notwendigkeit der Dauergenehmi-

34 BArch DP1 VA 3561 (Dokumente zur Taetigkeit des Bueros Gentz, 1974-76).

35 Viele Dokumente in dieser Mappe tragen den Vermerk: Wieder in den Panzerschrank.

36 BArch DP1 VA 3516 (Vermerk über eine Aussprache mit Rechtsanwältin und Notarin Ingeborg [sic] Gentz 8. 1. 1974) 
gung für die Ausreise nach Westberlin sei sie „gereizt“ geworden und habe wörtlich gesagt: „Was Vogel und Kaul Recht ist, ist mir billig. " 37

Am 24. Januar 1974 legte Ingeburg Gentz die bei dieser Gelegenheit vom Minister der Justiz angeforderte Aufstellung ihrer 1972 und 1973 aus notarieller und anwaltlicher Tätigkeit (BRD, USA, Schweiz) für DDR-Banken erzielten Deviseneinnahmen vor. In der Gesamtsumme von DM 2.487.899,64 (am folgenden Tag zu DM 2.488.399,64 korrigiert), so hob sie hervor, seien nicht enthalten die Überführung von Schmuck, Edelmetallen und Edelsteinen, „die ebenfalls unserer Wirtschaft zugute kamen“. ${ }^{38}$ Mutter und Sohn bekamen schließlich das gewünschte Dauervisum für 1974. Jedoch blieben ihre Pässe beim Ministerium der Justiz, und jeder Reisezweck hatte vorher geklärt zu werden.

Die vom Ministerium der Justiz angelegte Mappe zum Büro Gentz geht bis Ende 1975, als sie ihre Auslandstätigkeit aus Gesundheitsgründen bereits ganz hatte aufgeben müssen. Da ihr gesamtes Archiv in ihrem Keller verwahrt wurde (und, so Sabine Herrmann 2007, wird) und das Ministerium der Justiz schon aus Raumgründen eine Übernahme nicht verantworten konnte, ${ }^{39}$ erfolgte 1976 nicht nur die Bestellung ihres Sohnes zum Notar, sondern - noch erstaunlicher - nach seinem plötzlichen Tode kurz vor der Wende auch die Bestellung ihrer Enkelin Ute, die damals gerade ihr juristisches Staatsexamen abgelegt hatte. Die brisanten Akten im Keller des Gentz'schen Hauses machten es möglich - mit der Absegnung des Ministers der Justiz.

Ingeburg Gentz, eine hervorragende Juristin und starke, erfolgreiche und ehrgeizige Frau zählt zu den wenigen, die in einer zukünftigen Geschichte des Notariats der DDR einen besonderen Platz verdienen.

\section{Sabine Herrmann}

Die zweite Notarin, auf die dies zutrifft (wenn auch wohl in geringerem Maße), ist Sabine Herrmann, die als Notarinstrukteurin in Berlin in den Sechziger- und Siebzigerjahren auch bei Einzelnotar/inn/en, darunter Ingeburg Gentz, Revision zu machen hatte. ${ }^{40}$ Geboren 1933, war sie mit ihrer Familie aus Pommern geflüchtet und in Neubrandenburg untergekommen. Die Wirren der Kriegszeit hatten ein Abitur unmöglich gemacht. Kostenlose Abendkurse in der Volkshochschule in Buchhaltung, Stenografie und Schreibmaschineschreiben (1948-52) erlaubten erste Schritte in ein bescheidenes Berufsleben. Eine Bewerbung um einen Platz für die Schnellausbildung für Richter schlug fehl, da ihr Vater als Intellektueller eingestuft wurde. Dagegen gelang die Aufnahme in den dritten und letzten Lehrgang zur Ausbildung von Notar/inn/en an der Justizschule in Ettersburg bei Weimar (1956-57). Das zahlenmäßige Verhältnis zwischen Frauen und Männern „war etwa gleich“. Sie wurde Schülerratsvorsitzende, bestand als eine von 53 erfolgreichen Teilnehmer/inne/n (von anfangs 89) ihre Abschlussprüfung und wurde ab 1. März 1957 als Notarin im Staatlichen Notariat Potsdam/Stadt eingesetzt. ${ }^{41}$ Zu ihrer Enttäuschung erfuhr sie, dass Jurist/inn/en nicht mit der S-Bahn durch Westberlin fahren durften (sie hatte sich auf gelegentliche Kinobesuche dort gefreut), sondern eine extra dafür eingerichtete Umgehungsbahn zu benutzen hatten. Nach etwas über einem Jahr als Notarassistentin wurde sie „voll reingeschmissen".

1960 heiratete sie Dr. Ernst Herrmann, damals Verwaltungsdirektor an der Akademie in Babelsberg mit 2500 Villen von UFA-Schauspielern. Sie selbst wurde vom Direktor zur Beurkundung von Krediten für Reparaturen an den Villen herangezogen. 1964 wurde das Ehepaar nach Berlin versetzt, er an die Akademie der Wissenschaften, sie als Notarinstrukteurin am Stadtgericht Berlin. Von 1965 bis 1970 absolvierte sie „im Lumpensammlerverfahren“ (d.h. ohne Aufnahmeprüfung) das Fernstudium an der Humboldt-Universität. „Irgendwann fing die Humboldt-Universität an, Frauen zu immatrikulieren, weil die Männer nicht wollten.“ Das bedeutete ein Jahr Gesellschaftswissenschaften, dann fünf Jahre Jura - all das neben ihrer täglichen Arbeit und dem Sorgen für ein Kind. „Kein Wunder, dass Frauen oft verzichteten.“ Endlich war sie Diplomjurist und hatte alle Fächer mit 1 oder 2 bestanden.

Instrukteur/inn/e/n waren die rechte Hand des Leiters des Staatlichen Notariats des jeweiligen Bezirks. Sie arbeiteten selbstständig. Sie suchten die Notar/inn/e/n ihres Bezirks auf, organisierten Tagungen und Schulungen. Jedes Jahr gab es in Berlin eine große Tagung mit einer Ansprache des Ministers der Justiz, an der alle Notarinnen und Notare der DDR teilnahmen. Sie genoss es! Schon deshalb, weil sie der einzige weibliche Notarinstrukteur der Republik war. Und doch:

„Es war eigentlich Verwaltung. Wir machten Revisionen, mussten dann Notare runtermachen. Man vergaß alles, was man als Notar gelernt hatte, z. B. an der Reiseschreibmaschine ganz schnell Grundstücksverträge machen. “

Auf ihre Initiative hin wurden alle Berliner Notariate zusammengelegt und in jedem Stadtbezirk stattdessen eine Außenstelle eingerichtet. So brauchte man keinen Notarinstrukteur mehr, sondern lieber einen Leiter des Staatlichen Notariats Berlin - Sabine Herrmann. Sie blieb es bis 1985 . Ihre Expertise war schon seit den 1970er-Jahren so anerkannt, dass sie als einzige Frau eingeladen wurde, an dem lange geplanten, am 1. Februar 1982 endlich erschienenen Handbuch des Notariats mitzuwirken.

Mit ihrer Ernennung zur Leiterin der Außenstelle des Staatlichen Notariats Berlin im Spezialbereich internationales Erbrecht begann eine weitere Phase ihres Berufslebens - der Schritt in die Öffentlichkeit. Sie übernahm Lehrtätigkeiten,

37 Die Rechtsanwälte Prof. Dr. Wolfgang Vogel (1925-2008) und Prof. Dr. Friedrich Karl Kaul (1906-81) gehörten zu der kleinen Zahl von Anwaltsnotaren, die wie Dr. Ingeburg Gentz auch in Westberlin zugelassen waren und dort die Interessen der DDR vertraten.

38 BArch DP1 VA 3561

39 BArch DP1 VA 4791 (Hausmitteilung im Ministerium der Justiz, 18.10.1978).

40 Informationen zu Sabine Herrmann entstammen einem ausführlichen Gespräch mit ihr (9.5.2007) sowie Kommentaren früherer Kolleginnen.

41 Genaue Angaben zu Inhalt und Durchführung dieses Lehrgangs finden sich bei Osterburg, op. cit., S. 140-45. 
hielt Vorträge, veröffentlichte regelmäßig in Tageszeitungen zu Leserfragen. Bis 1990 war sie es, die als Notarin beim Fernsehen der DDR bei der Ziehung von Lottozahlen und im Telelotto Beurkundungen vornahm. In 33 Fernsehunterhaltungssendungen von „Wennschon-Dennschon“ war sie als Juryvorsitzende tätig. ${ }^{42}$ Alle in der DDR kannten sie.

Zunehmend wurden GmbHs und AGs gebraucht, weil unter dem Namen „Volkseigentum“ jede volkseigene Fabrik für jede andere gepfändet werden konnte. In dem „weißen Haus am Bahnhof Friedrichstraße (da saßen sie alle, z. B. Schalck-Golodkowski mit seiner Kunst- und Antiquitäten$\mathrm{GmbH}$ )“ musste beurkundet werden. Ihr fiel sozusagen spiegelverkehrt die Rolle zu, die am Anfang der DDR Ingeburg Gentz innehatte.

"Das Ministerium kam zu mir. Ich hatte nie eine GmbH gesehen oder begründet. Nur ein alter Rechtspfleger in Berlin konnte das noch. Das Ministerium konnte einem noch nicht einmal die Formularbücher aus dem Westen besorgen. "

So ging sie zu dem Rechtspfleger, kündigte eine Revision an, nahm die Verträge zur GmbH-Gründung mit und kopierte sie. Ergänzt durch etwas Literatur aus der Humboldt-Universität wurde das die Grundlage für „Beurkundungen im Handelshaus“. Dann kam die Wende, und ihre gerade erworbenen Kenntnisse trugen ungeahnte Früchte. Ganze VEBs mussten bis zur Währungsunion am 1. Juli 1990 in GmbHs umgewandelt werden. An zwei Wochenende bewältigte sie das Schicksal der gesamten Schuhindustrie der DDR.

\section{Rückblick}

Die winzige Berufsgruppe der Notar/inn/e/n - zum Zeitpunkt der Vereinigung waren es insgesamt 379, davon 257 Frau- $e^{43}$ - wird nie im Rampenlicht der DDR-Justizgeschichte bzw. deren Justizgeschichtsschreibung stehen. Es bedurfte des sorgfältigen Nachforschens vermittels verstreuter ArchivDokumente, vor allem aber persönlicher Gespräche mit den damals selbst Beteiligten, um über bloße statistische und andere offizielle Angaben hinaus dem Wesen dieses Notariats nachzuspüren. Die Frauen, die sich zu solchen Gesprächen bereiterklärten (sowohl solche, die nach 1990 den Schritt in die Selbstständigkeit gewählt hatten, als auch andere, ältere, deren Berufsleben mit dem Ende der DDR zusammenfiel) berichteten keineswegs unkritisch, aber mit Engagement und Wärme von ihrer Tätigkeit. Gemeinsamer Nenner waren die (relative) Selbstständigkeit bei der Arbeit sowie das Gefühl, dass diese Arbeit trotz politisch vorgegebenem engen Rahmen den Rat suchenden Bürgerinnen und Bürgern „etwas brachte“. Arbeits- und Lernwille sowie fürsorgliches Interesse an den Sorgen der Mitbürger, nicht aber herausragende juristische Fähigkeiten waren bei der Ausübung ihres Berufs gefragt. Desto stärker beeindrucken Persönlichkeit und Leistung der hier je einzeln vorgestellten Notarinnen Dr. Ingeburg Gentz und Sabine Herrmann.

42 Herrmann, Sabine, in Huebner, Ralph (Hrsg.), Who Is Who in der Bundesrepublik Deutschland. Supplementwerk der biographischen Enzyklopädie führender Frauen und Männer Deutschlands, Verlag für Personenenzyklopädien 1998.

43 Bundesnotarkammer, Notarverzeichnis der Bundesländer Ost 1990.

\section{Rechtsanwältinnen in der DDR}

\section{Dr. Stefanie Loroch}

Münster

Entgegen manch düsterer Prophezeiung ${ }^{1}$ entpuppte sich die Zulassung der Frauen zur Anwaltschaft vor 90 Jahren keineswegs als Schaden, vielmehr als Segen für die Rechtspflege. Justizrat Dr. Bieber, der 1922 anlässlich der 14. Vertreterversammlung der Anwaltschaft die vermeintliche Nichteignung der Frau zum Juristenberuf schlicht als Vorurteil abtat, behielt Recht. ${ }^{2}$ Die Geschichte hat die Gegenargumente von einst überholt: Justitia ist eine Frau und der Rechtsanwalt immer häufiger weiblich.

Fast ein Drittel der zugelassenen Rechtsanwältinnen und Rechtsanwälte sind nach der im Jahr 2011 veröffentlichten Statistik der Bundesrechtsanwaltskammer Frauen, Tendenz steigend. ${ }^{3}$ Dies ist ein nicht zu unterschätzender Wert, denn der Zeitraum seit Zulassung der ersten Rechtsanwältin im Jahr 1922 ist überschaubar kurz.
Der Nationalsozialismus beendete vorläufig, was gerade erst begonnen hatte. Nach dem Ende des zweiten Weltkrieges begann eine Phase des Wiederaufbaus und der politischen Umstrukturierung. Die mit der Einteilung in die Besatzungszonen einhergehende Rechtszersplitterung bedingte letztlich ein Auseinanderdriften der Anwaltschaft im Ostteil und im Westteil Deutschlands. Damit nahm auch die Geschichte der Rechtsanwältinnen in der BRD und der DDR einen unterschiedlichen Verlauf.

\section{Der weibliche Rechtsanwalt in der DDR}

„Sie geht davon aus, daß sie als ausgebildeter Jurist einen Anspruch darauf hat, ihren persönlichen Wünschen entsprechend

\footnotetext{
1 Vgl. Protokoll der 14. Vertreterversammlung der Anwaltschaft 1922, in: JW 1922, S. 1246 ff.

2 Protokoll der 14. Vertreterversammlung der Anwaltschaft 1922, in: JW 1922, 1246 ff. (S. 1247-1249).

3 Stand 1.1.2011: 32,04\%.
} 